\title{
Synthesis of Ni-rich cathode material from maleic acid-leachate of spent lithium-ion batteries
}

Borui Liu ${ }^{\dagger}$, Qing Huang ${ }^{\dagger *}$, Yuefeng Su ${ }^{\dagger *}$, Liuye Sun ${ }^{\dagger}$, Tong $\mathrm{Wu}^{\dagger}$, Qiyu Zhang ${ }^{\dagger}$, Guange

Wang ${ }^{\dagger}$, Feng $W u^{\dagger}$

$\uparrow$ School of Materials Science and Engineering, Beijing Institute of Technology, China, 100081

\section{Corresponding Author}

* Q. Huang. Email: huangqing3121@sina.com; Telephone: 86-10-6818099;

* Y. Su. Email: suyuefeng@bit.edu.cn Telephone: 86-10-6818099;

Number of pages : 6

Number of figures: 6

Number of tables: 1 


\section{Support information}
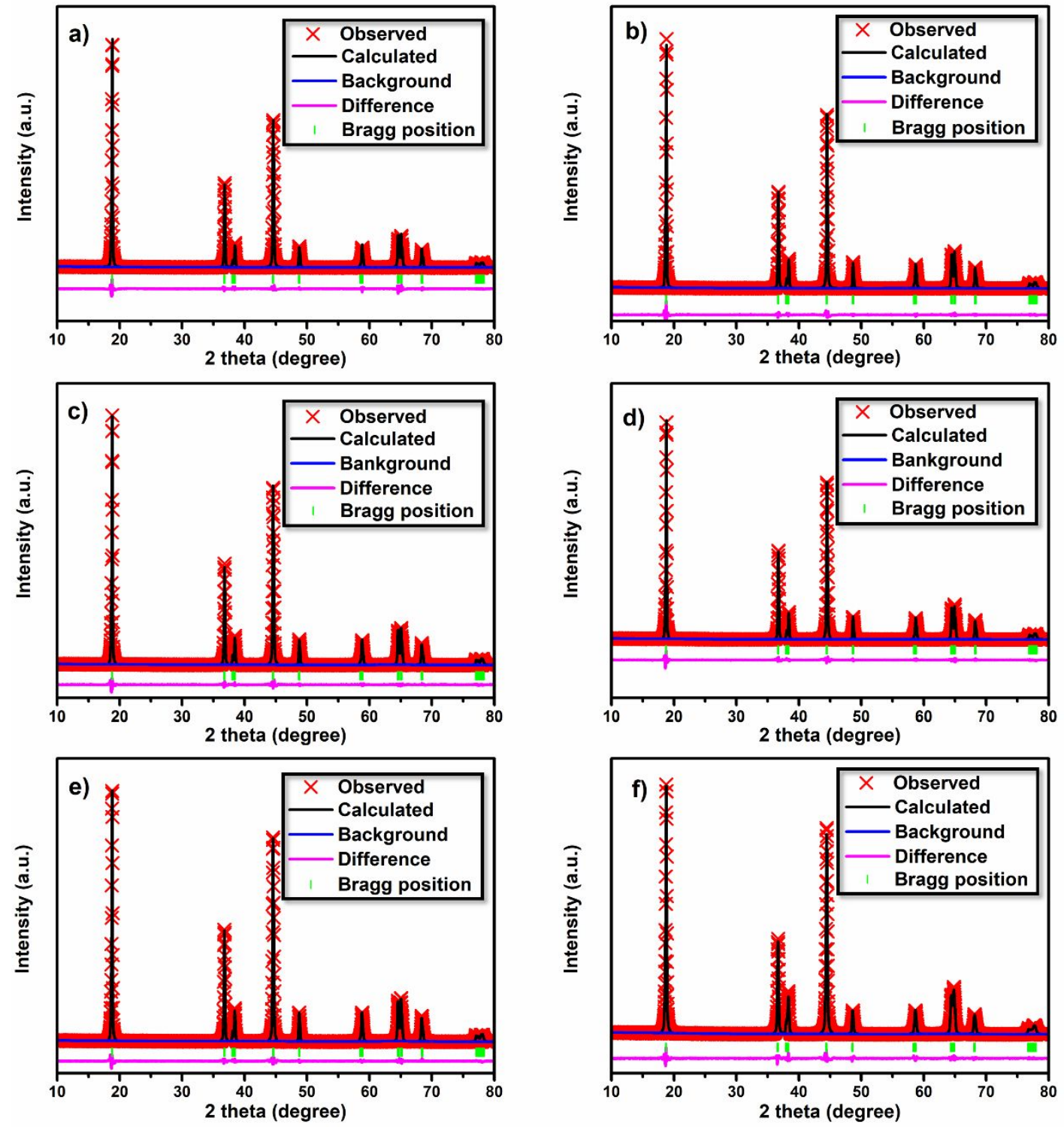

Figure S1. Rietveld refinement patterns of NCM-0 (a), NCM-20 (b), NCM-40 (c), NCM-60

(d), NCM-80 (e), NCM-100 (f), materials in the experiment 

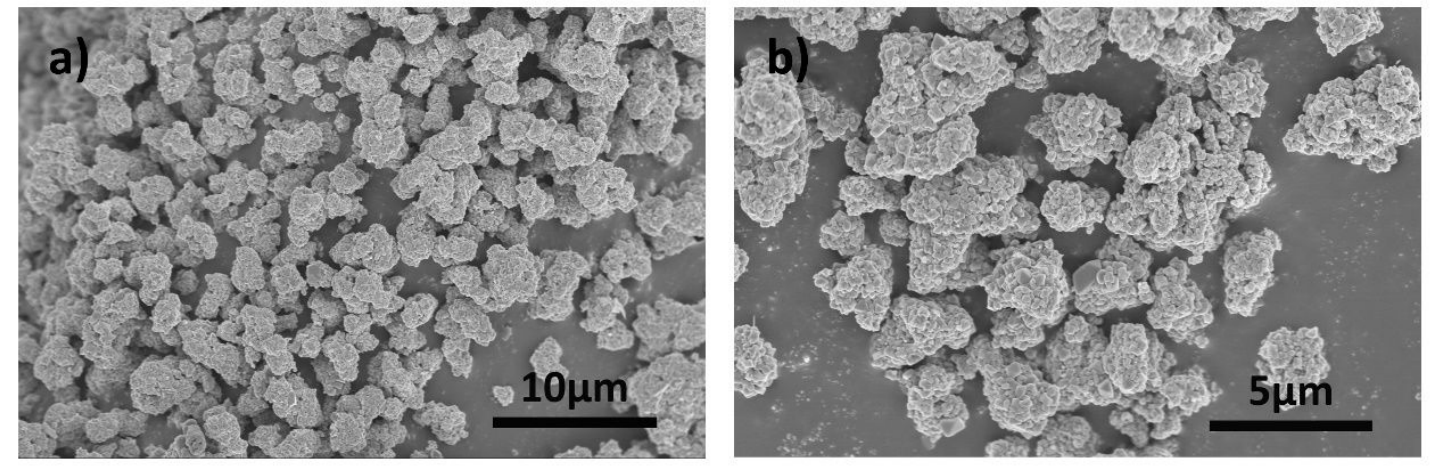

Figure S2. The SEM image of NCM-80 (a) and NCM-100 (b) materials
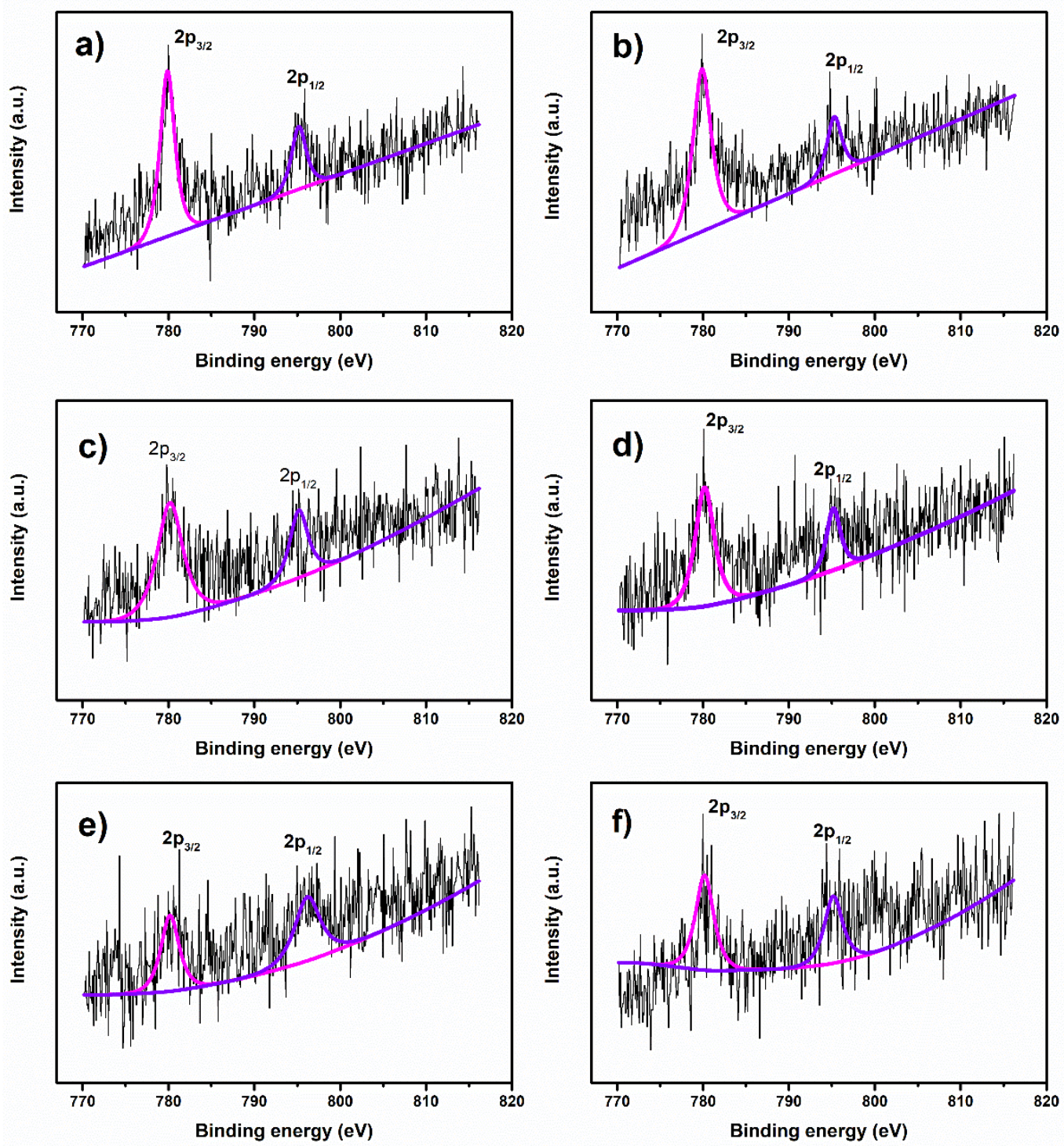

Figure S3. XPS spectra of Co 2p of the NCM-0 (a), NCM-20 (b), NCM-40 (c), NCM-60 (d), 

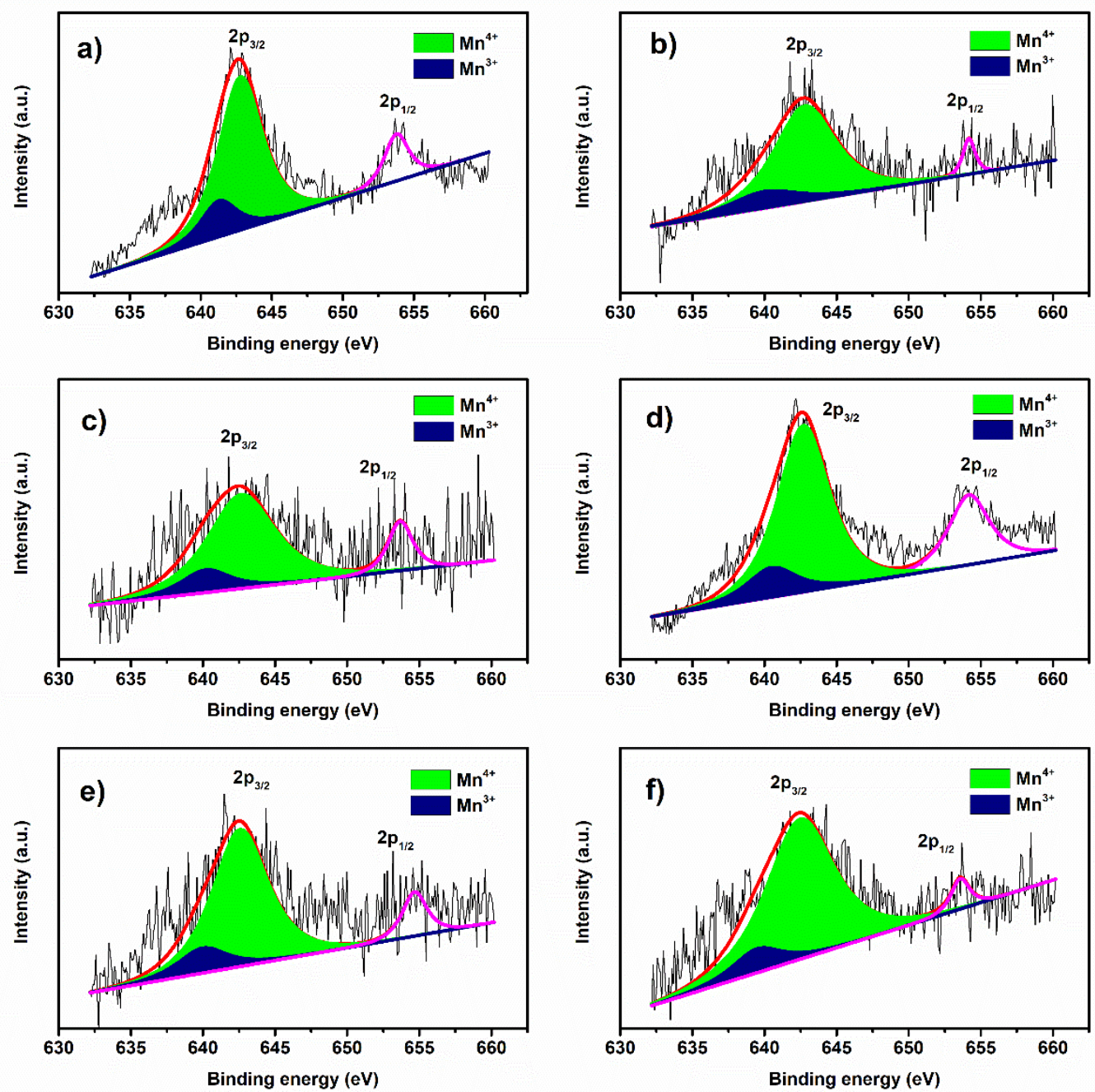

Figure S4. XPS spectra of Mn 2p of the NCM-0 (a), NCM-20 (b), NCM-40 (c), NCM-60 (d), NCM-80 (e), NCM-100 (f) materials 

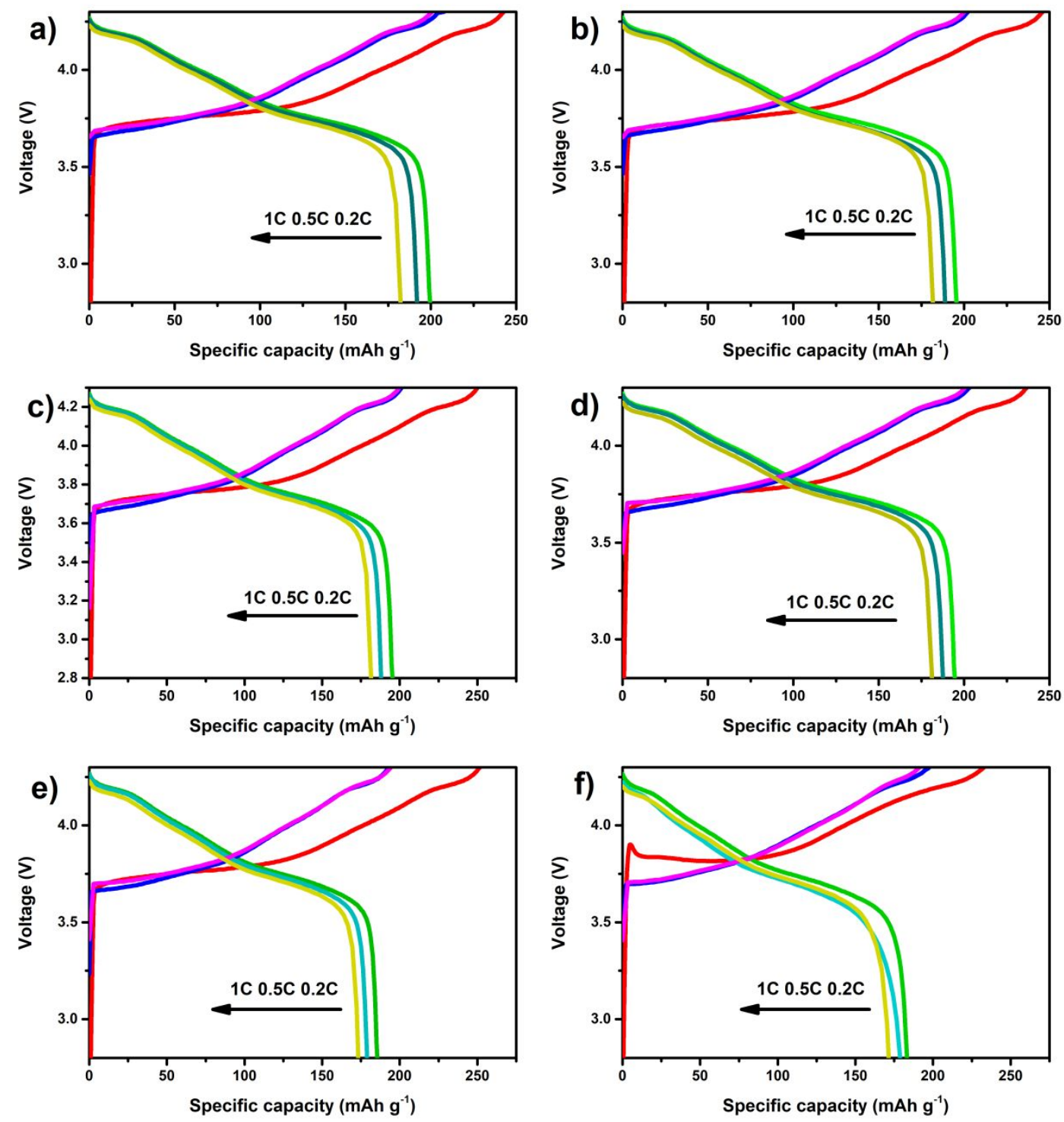

Figure S5. The initial charge-discharge curves of NCM-0 (a), NCM-20 (b), NCM-40 (c), NCM-60 (d), NCM-80 (e), NCM-100 (f) at $0.2 \mathrm{C}, 0.5 \mathrm{C}$ and $1 \mathrm{C}(2.8$ to $4.3 \mathrm{~V})$ 

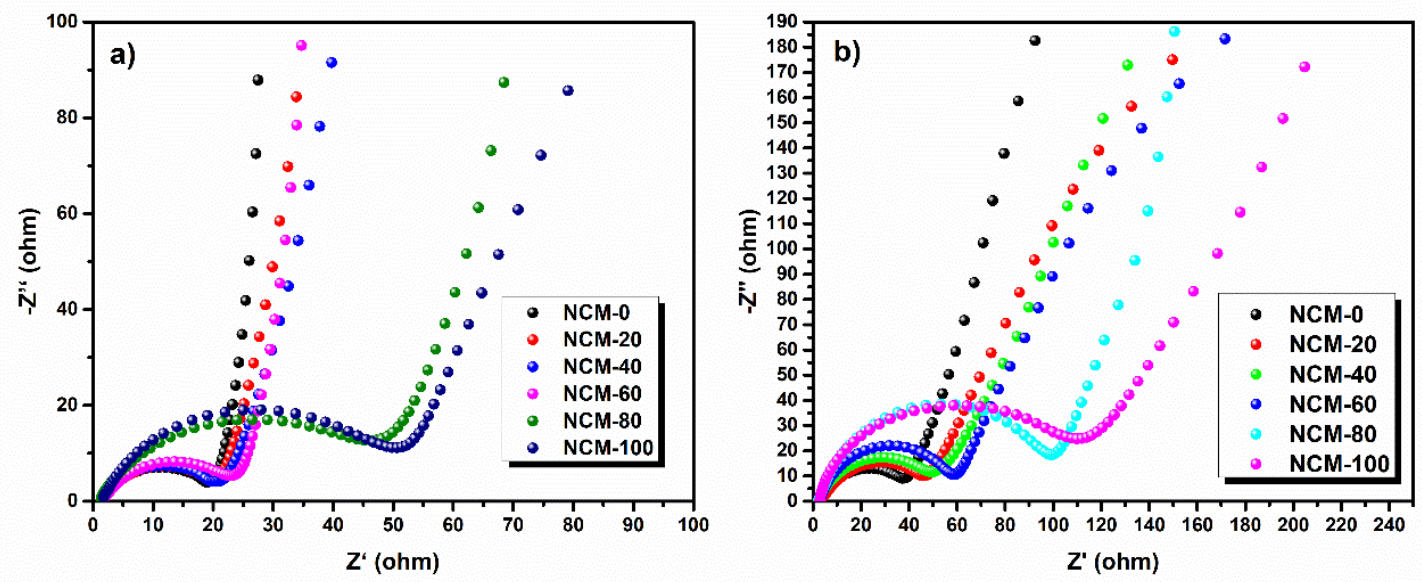

Figure S6. Nyquist plots of the six NCM materials in the experiment after the 1st (a) and 100th (b) cycle at $1 \mathrm{C}$

Table S1. The $\mathrm{Ni}^{2+} / \mathrm{Ni}^{3+}$ ratios in the six $\mathrm{NCM}$ materials

\begin{tabular}{ccccccc}
\hline No. & NCM-0 & NCM-20 & NCM-40 & NCM-60 & NCM-80 & NCM-100 \\
\hline $\mathrm{Ni}^{2+} / \mathrm{Ni}^{3+}$ & 0.26 & 0.464 & 0.480 & 0.514 & 0.649 & 0.798 \\
\hline
\end{tabular}

